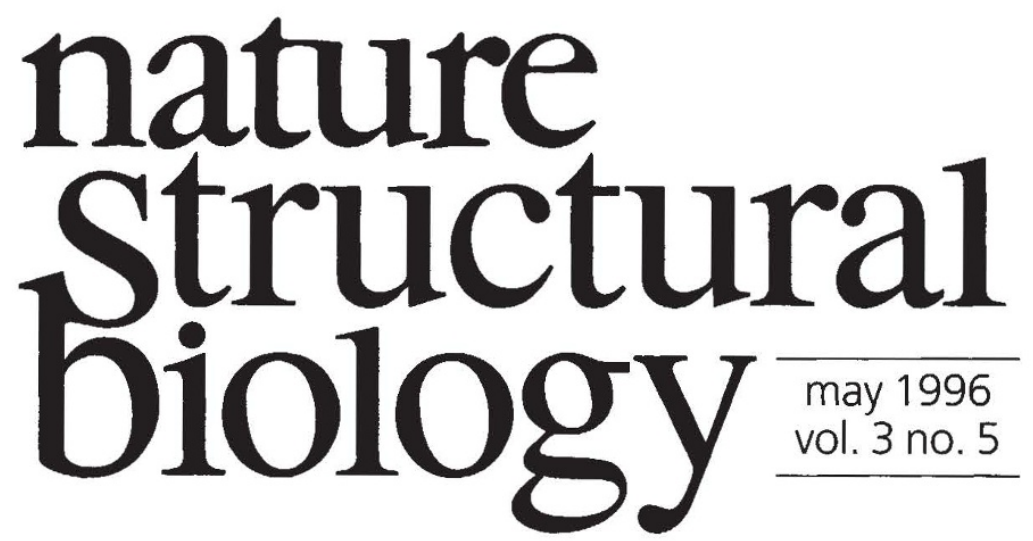

\title{
Membrane proteins in the USA
}

There is an oft heard claim - made mainly by Europeans - that the only serious work carried out on the atomic resolution structures of membrane proteins is done outside the USA (and mainly in Europe). This has much more foundation in reality than it should, given the level of funding and scientific skills available in the States. In an attempt to tip the balance in favour of the USA the National Institutes of Health (NIH) - the US Government funding agency for biological and medical sciences-are attempting to affect a sea-change in funding patterns (and attitudes to risky science) that will actively encourage the study of membrane protein structure in the States.

The importance of divining the structure and function of membrane proteins is fundamental to our understanding of biology: membrane proteins are the means by which all cells communicate and interact with the surrounding environment. The difficulty of handling these highly hydrophobic proteins has made determining their structures extremely challenging, a problem further exacerbated by the fact that the methodologies for structure determination using X-ray crystallography (and more recently NMR spectroscopy) have been developed for water-soluble globular proteins. But scientists working in the US are no less interested in taking on the challenge than their counterparts in the rest of the world. So why is the United States so poorly represented-relative to the size of the basic research base-in the pantheon of solved membrane structures? The root cause of the problem seems to be in the funding mechanisms that underlie the research effort in the US and the effect these have on the 'sociology' of scientific research.

The US system is geared to rewarding science that has a high probability of being successful in the short-to-medium term (three to five years). Indeed, as many problems can be quantized into a series of incremental advances-that

"There comes a time when [molecular biological, biophysical and biochemical] studies start to provide exponentially diminishing returns and you have to determine the structure [of membrane proteins] to really understand function" -Rod MacKinnon define both the minimum publishable unit and the average post-doctoral stintthe system has proven to be highly effective. But not all areas of research can be dispatched in such a safe and conveniently packaged manner: the study of the atomic structure of membrane proteins is one of them.

It is in such areas that the American system suffers at the hands of its own success. The standards of the preliminary data required to achieve a successful grant application - the informal 'no crystals, no grant' or, for NMR spectroscopy 'no spectra, no grant'- -would have, until recently, automatically excluded almost all membrane protein structure applications. Thus, proposals to study membrane protein structure have been slipped in as part of grant applications for more 
"The problem of judging all such blue-sky research is weighing potential impact against the probability of success" Richard Henderson 'run-of-the-mill' projects. Furthermore, grants in the US are rarely longer than five years in duration, with renewal being strictly dependent on material evidence of progress, measured in published papers. Looking to Europe and Japan it is clear that stable long term funding (on the order of decades) with relatively few strings attached (by US standards) has played an essential role in encouraging membrane structure research there.

Worse still, the process of tackling membrane structure is more often akin to cookery than basic science and the pay-off of solving the structure tends to be concentrated into a relatively small number of publications at the end of what may be many years of work. Such an outcome is essentially incompatible with career advancement in the context of the US 'publish or perish' system. Thus, much of membrane protein structural biology has been limited to 'chipping away at the edges' using molecular biological, biophysical and biochemical techniques. But, as Rod MacKinnon, presently at Harvard University, Massachusetts, observes, "there comes a time when such studies start to provide exponentially diminishing returns and you have to determine the structure to really understand function".

In an attempt to encourage scientists in the US to do just this, the NIH launched a program announcement (PA, in the jargon) at the beginning of last year-entitled Structural Biology of Membrane Proteins - directed at research into every step on the way to obtaining atomic resolution structures of membrane proteins. While there has been a small amount of interest generated by the PA (30-40 application so far), there has also been a certain amount of confusion among those charged with reviewing the applications (so-called study sections) about the way in which the PA should be implemented.

Part of the problem rests with the fact that there is no specific amount of money set aside for the initiative: applications sent in under the auspices of the PA must compete with the general pool of applications. Peter Preusch, Program Director, Division of Pharmacology, Physiology and Biological Chemistry at the $\mathrm{NIH}$, and one of the main authors of the PA, argues that they wished to "avoid trying to specifically direct research" and therefore chose not set aside a pot of money to support the initiative. "The amount of money the PA can attract will depend solely on the strength of the applications", he says. This has not been completely true. John Norvall, Assistant Director for Research Training, National Institute for General Medical Science at the NIH, notes that of the $~ 7-10$ applications funded under the auspices of the PA several initially refused funding were later 'rescued' by lowering the cut-off point. Norvall is also hopeful that a number of the applications that were declined this time around will be more successful on a second submission, once they are suitably revised.

Comparing the necessarily more preliminary membrane protein applications (where success is not guaranteed) with those of much more tractable systems (where success is essentially assured) seems to have presented problems for the study section reviewers. "It's a little like trying to compare apples and oranges" one study section reviewer complains. Certainly, separate criteria and a separate pot of money would have made the reviewers' job an easier one. "The problem of judging all such blue-sky research is weighing potential impact against the probability of success," notes Richard Henderson, of the Laboratory of Molecular Biology, Cambridge. But the NIH funding system "does not seem to be set up to evaluate proposals for risky research," Henderson adds. Nonetheless, both Norvall and Preusch seem to believe that just such decisions can be made using the present review criteria. Thus the challenge for the study sections is to throw off the 'no crystal, no grant' blinkers and back risky and exciting research. 